\title{
Perceptual grouping measured by color assimilation: Regularity versus proximity
}

\author{
Rob van Lier *, Johan Wagemans \\ University of Leuven, Department of Psychology, Tiensestraat 102, B-3000 Leuven, Belgium
}

Received 19 February 1997; accepted 7 April 1997

\begin{abstract}
Two powerful grouping principles in visual perception have been studied rather frequently, namely, regularity and proximity. In many cases, however, the impact of either regularity or proximity was examined. The goal of the present research is to provide and test an experimental paradigm on the basis of which the relative strengths of these grouping factors can be studied. The proposed paradigm is based on early Gestalt observations that colors of local elements within a perceptual whole tend to look more like each other than they actually are. Three experiments were set up to test whether this phenomenon of color assimilation can provide a means to compare perceptual groupings induced by regularity and proximity. In all experiments, the stimuli were linear configurations of colored elements with variable distances between the elements. The task of the participants was to compare the color of a single test element with the colors of two different groups of elements. In Experiment 1, for all colors, the color of the test element was judged to be more similar to the color of the elements with which it can be grouped by means of regularity. In Experiment 2, for specific colors, the color of the test element tended to be more similar to the elements with which it can be grouped by means of proximity. Finally, in Experiment 3, configurations with a greater variety of positions were tested, including those yielding conflicting groupings, that is, on the basis of regularity and proximity different groupings were to be expected. The color judgements for these stimuli clearly reflected the competition between the two factors. (C) 1997 Elsevier Science B.V.
\end{abstract}

PsycINFO classification: 2323

\footnotetext{
* Corresponding author. Tel.: +32 163260 18; fax: +32 163260 99; e-mail: robert.vanlier@psy.kuleuven.ac.be.
} 
Keywords: Perception; Vision; Form and shape perception; Color perception

\section{Introduction}

Ever since perceptual phenomena have been studied, the mechanisms and rules of perceptual grouping have received considerable attention. Every student of perception is familiar with the various perceptual laws that have been discovered by early Gestalt psychologists (e.g., Koffka, 1935; Köhler, 1920; Wertheimer, 1923). To explain perceived interpretations, they formulated various so-called Gestalt laws such as proximity, similarity, good continuation, or closure. It appeared, however, that it was virtually impossible to predict the perceptual outcome in patterns which give rise to multiple groupings following different principles. Mainly for this reason, Palmer and Rock (1994) announced the demise of Gestalt psychology. Nevertheless, contemporary theorizing on perceptual grouping, and visual perception in general, often can be traced back to Gestalt psychology. For example, the current widely known Recognition-By-Components theory on object perception (Biederman, 1987), as well as various approaches within the field of computer vision (e.g., Lowe, 1985), are partly based on grouping principles proposed by Gestalt psychologists.

In this paper, we focus on two powerful grouping principles that have been the subject of intensive research. The first is the notion of Good Form, or Prägnanz, or regularity, as formulated by Koffka (1935). The notion of Prägnanz can be considered as the precursor of the global-minimum principle as formulated by Hochberg and McAlister (1953), which states that the simplest interpretation of a pattern will be selected by the visual system. The so-called Structural Information Theory (SIT) initiated by Leeuwenberg (1969) and further elaborated in more recent work (e.g., Van der Helm and Leeuwenberg, 1991, 1996; Van Lier et al., 1994, 1995) is based on this global-minimum principle. Within SIT, a set of regularity-based coding rules and its quantification of regularity allow a precise testing of the theoretical predictions for a broad range of patterns, ranging from series to surfaces and objects (e.g., Van Lier, 1996).

The second grouping principle which has been attracting considerable attention recently, is proximity. Elaborate attempts have been made in investigating grouping based on proximity (e.g., Kubovy and Wagemans, 1995; Van Oeffelen and Vos, 1983). Kubovy (1994) and Kubovy and Wagemans (1995) presented a theoretical and experimental framework by means of which the organizational impact of proximity can be tested in dot lattices. The authors showed that at least with respect to dot lattices, perceptual organization can be described very well by means of proximity alone. In their modelling of the perception of dot lattices, each dot is attracted to its neighboring dots. The force of attraction declines as an exponential decay function of distance. The authors demonstrated that despite the various differences in the configurational regularities between the dots in the lattices, the distance-based decay function suffices for the explanation of their data (see also Kubovy et al., 1996). 
So, whereas regularity-based theories such as SIT focus on structural aspects, proximity-based theories such as those of Kubovy and Wagemans (1995) principally focus on metrical aspects. Both approaches clearly state the superiority of the factor they operationalized (regularity and proximity, respectively), but they do not deny the possible impact of the other grouping factor (proximity and regularity, respectively). Facing the empirical success of these contemporary approaches, they somewhat ironically lead us back to an old Gestalt-like question: Given a certain visual pattern, which of the grouping principles is stronger, regularity or proximity?

In this paper, we re-emphasize the importance of both regularity and proximity and introduce a method to demonstrate and measure the relative influences of these tendencies. These tendencies may co-operate with each other, that is, support the same perceptual grouping, or compete with each other, that is, support different perceptual groupings.

Consider the simple dot patterns in Fig. 1. Both patterns consist of five dots, labelled A-E. Let us focus first on D in Fig. 1(a). The issue of perceptual grouping can be rephrased as an issue of "belongingness": To which of the other dots of the pattern does D belong most strongly, to A, B, C, or E? As the distance between D and E is smaller than the distance between $D$ and any of the other dots, $D$ is likely to be grouped with $\mathrm{E}$. Moreover, the other dots (A, B, and C) might be grouped together as they constitute a perfect isosceles triangle. So, in Fig. 1(a), regularity and proximity support the same belongingness of $\mathrm{D}$.

In Fig. 1(b), D has been shifted towards E, and away from A, B, and C. Clearly, on the basis of proximity alone, the grouping between $\mathrm{D}$ and $\mathrm{E}$ should be stronger than in Fig. 1(a), whereas the grouping with A, B, and C, should be weaker. Nevertheless, due to the special position of $\mathrm{D}$, constituting a square with $\mathrm{A}, \mathrm{B}$, and $\mathrm{C}, \mathrm{D}$ appears to be grouped more strongly with $\mathrm{A}, \mathrm{B}$, and $\mathrm{C}$, whereas the grouping with $\mathrm{E}$ tends to be less strong. In contrast with Fig. 1(a), the grouping in Fig. 1(b) is more ambiguous: The different groupings seem to compete with each other. Answering the question to which of the dots $\mathrm{D}$ belongs most strongly, certainly is not a simple task.

\subsection{Measuring perceptual grouping}

Perceptual grouping has been investigated by several researchers during past decades, often focusing either on regularity or on proximity. For example, Van der Helm et al. (1992) tested the perceptual grouping by regularity within a series of symbols by means of pairwise comparisons between different clusterings of the symbols within that series. In their experimental set-up, however, the stimulus set was restricted such that only grouping due to various types of regularities, like bilateral symmetry or alteration, could be examined. Given their theoretical framework and their test of assumptions within that framework, such a strategy was legitimate but their claims presumably cannot be extrapolated and generalized straightforwardly to patterns in which proximity plays a more evident role.

Other investigations focus on proximity (e.g., Kubovy and Wagemans, 1995; Oyama, 1961). In Oyama's experiments (Oyama, 1961), grouping in one direction of a rectangular dot lattice was pitted against grouping in another direction. It appeared 
A

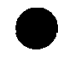

B

\section{A}

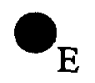

D

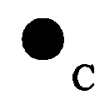

A

B

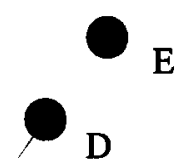

$\mathrm{C}$

B

Fig. 1. The competition between grouping by regularity and proximity. In pattern A, Dot D is most likely to be grouped with Dot $\mathrm{E}$ because of proximity, whereas Dots $\mathrm{A}, \mathrm{B}$, and $\mathrm{C}$ constitute an isosceles triangle. In pattern $B$, Dot $D$ has been shifted towards Dot $E$, and away from Dots $A, B$, and $C$. Nevertheless, the grouping is more ambiguous as Dot $D$ now also seems to be grouped with Dots $A, B$, and $C$, because of the formation of the square $\mathrm{A}, \mathrm{B}, \mathrm{C}$, and $\mathrm{D}$.

that the total time that observers reported either columns or rows, that is, the relative strength of perceptual grouping, varied as a power function of the relative spatial separation. However, in line with arguments given by Rock and his co-workers (Girgus et al., 1977; Kubovy et al., 1997; Rock and Mitchener, 1992) they criticized the experimental set-up because of the fact that the reversibility of figures depends strongly on the awareness of the observers on the possibility of reversibility. To cope with such problems, Kubovy and Wagemans (1995) proposed a procedure in which dot lattices were presented for short periods $(300 \mathrm{~ms})$. After each presentation, the observer was asked to decide on the perceived orientation of the lattice. In spite of the different configurational properties of the lattices, the results could be interpreted on the basis of proximity alone. Their design, however, did not allow an examination of the predictions derived from regularity-based theories. Doing so seems to be a hazardous enterprise as regularity-based models may not directly relate config- 
ural properties with perceived direction. Furthermore, it can be questioned if the configural properties of the dot lattices are salient enough to compete with proximity as a grouping factor. Future research using a broader range of patterns may answer the generalizibility of their proximity-based model.

Clearly, all of the above experimental techniques are restricted to specific stimulus configurations. Our aim here is to provide an experimental method that could be employed to a variety of patterns. How could the perceptual grouping or perceptual belongingness of a single element, such as Dot D in Fig. 1, be determined? Of course, one possibility is to simply ask observers. An obvious problematic aspect is that such a method would be extremely sensitive to demand characteristics. A more indirect way to measure the perceptual group can be derived from the observation that elements that belong to the same group often tend to look more like each other than they physically are, a process that has become known as assimilation (e.g., Fuchs, 1923; Kanizsa, 1988; King, 1988; Musatti, 1931). Notice that it makes good ecological sense to have elements that belong together look more like each other: It facilitates the formation of strong perceptual units that are easier to segregate from their background. This figure-ground separation or grouping often has to work fast and efficiently on imperfect visual data (e.g., when an animal has to detect a predator partly occluded by a bush).

By means of an illustration, consider a well-known example of context-sensitivity in Fig. 2. It contains two numbers (12 and 14) and two letters ( $A$ and $C$ ) but the central symbol is somewhat ambiguous. In the context of the letters $A$ and $C$ (reading from left to right), it is readily interpreted as a letter $B$, whereas in the context of the numbers 12 and 14 (reading from top to bottom), we read the symbol as 13. A similar case of ambiguity to similarity, which is more directly related to the issue of the present paper, can be found in a series of experiments presented by the Gestalt psychologist Fuchs (1923).

\subsection{Perceptual grouping and color assimilation}

Fuchs (1923) described an intriguing phenomenon that could provide an instrument to measure perceptual belongingness of a single element. Consider one of $\mathrm{Fu}$ chs's examples in Fig. 3. The elements in the $3 \times 3$ square matrix of dots are yellow, red, or orange (represented by grey values). The orange dot in the middle appears to change its color as a function of perceptual grouping. If the orange dot is conceived

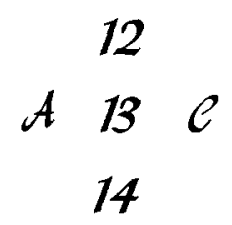

Fig. 2. The interpretation of the ambiguous central symbol depends on its context. When reading from left to right, the central symbol is readily interpreted as the letter $B$. When reading from top to bottom, the central symbol is most likely to be interpreted as the number 13 . 


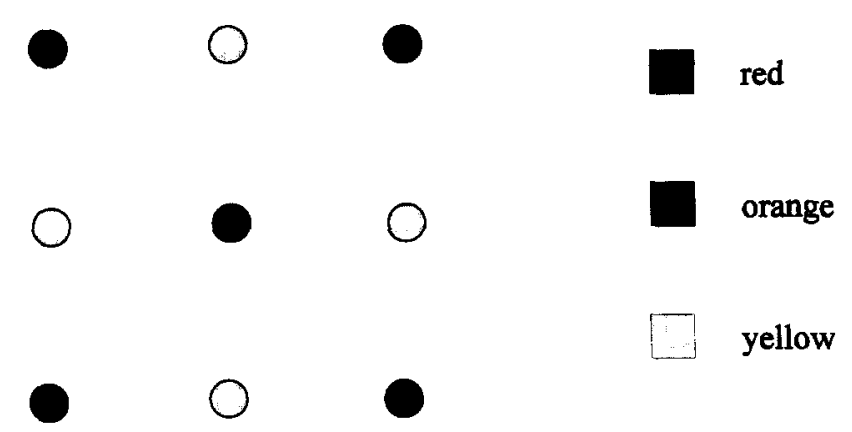

Fig. 3. One of the patterns investigated by Fuchs (1923), involving color assimilation. If the central orange dot is considered to be a part of the yellow cross $(+)$, the orange color appears to look rather "yellowish", whereas if the same dot is considered to be a part of the red cross $(x)$, it appears to look more "reddish". (Note that colors have been replaced by grey values as indicated in the legend.)

as an element of the yellow plus sign $(+)$ it appears to look more "yellowish", whereas the same orange dot appears to look more "reddish" if it is conceived as an element of the red cross $(x)$. This effect of color assimilation, originally observed by Von Bezold (1876), has been studied by Fuchs using various types of displays. Fuchs (1923) hypothesized that color assimilation was mediated by the formation of a perceptual whole.

During past decades the notion of assimilation within a perceptual whole has been discussed by several other researchers. For example, in reviewing a number of visual illusions, King (1988) concluded, in line with Fuchs, that they all could be explained by assuming that assimilation is due to the perception of one whole, whereas contrast is due to the perception of two wholes. In reply to King's paper, Kanizsa (1988) pointed out the relation between the "perceptual whole" hypothesis of Fuchs (1923) and King (1988), and Musatti's law of "maximal homogeneity" (Musatti, 1931). Kanizsa further argued that the idea of assimilation within a perceptual whole may serve as an explanation for other perceptual phenomena, like perceived transparency of surfaces (Kanizsa, 1979; Metelli, 1967). In contrast with this idea, De Weert (1991) and De Weert and Van Kruysbergen (1987) initially presented an explanatory model of assimilation based on local averaging on large receptive fields of center-surround cells in the retina. Recently, however, De Weert and Van Kruysbergen (1997) demonstrated assimilation effects that seem to be mediated by more "central" routes. One of their demonstrations suggests that assimilation occurs after the figure-ground segregation has taken place, a phenomenon that also has been highlighted by Festinger et al. (1970). These findings further indicate that assimilation occurs within a perceptual whole. In line with these findings, De Weert and Van Kruysbergen (1997) concluded that the explanation based on peripheral processes cannot account for all assimilation effects.

If perceptual grouping causes color assimilation, it provides a tool to investigate the perceptual belongingness of local elements in visual patterns like in Fig. 1. However, some precautions must be taken. One must avoid conditions in which regularity 
or proximity (or both) do not give rise to color assimilation, and may even induce contrast effects (e.g., DeValois and DeValois, 1988; De Weert, 1991). Contrast effects have been studied extensively in past decades and have received even more attention than assimilation effects, perhaps driven by the discovery of the mechanism of lateral inhibition (as argued by De Weert, 1991). This does not mean that, contrary to assimilation, contrast effects are mediated solely by peripheral mechanisms. There are several indications that also in case of contrast effects, both peripheral and central aspects play a role (e.g., DeValois and DeValois, 1988). For example, Benary (1924) and, more recently Agostini and Proffitt (1993), showed that, at least for achromatic stimuli, contrast effects can be evoked by perceptual grouping. Grossberg and Mingolla (1985) have provided a mechanistic explanation of many interesting phenomena related to assimilation and contrast in terms of their so-called "neural dynamics of form perception".

An essential condition for an unambiguous measurement of possible competitive effects of regularity and proximity is that their specific individual effects on color perception can be measured. Although color induction by regularity and proximity may follow different routes - central, peripheral or a mixture of both - these competitive causes can be compared rather straightforwardly if they have similar effects. Further, it should be noticed that the relation between color assimilation and grouping is not necessarily uni-directional. Previous research (e.g., Stalmeier and De Weert, 1988) showed that color assimilation can be used to disambiguate perceptual organization but what we try to do is the opposite. We assume perceptual grouping and measure induced color assimilation effects.

In the following experiments, we first investigate whether regularity and proximity induce similar effects for specific colors. In Experiment 1, the color induction due to regularity is examined in patterns in which possible influences of differences in proximity are minimized. In Experiment 2, the same is done to examine effects of proximity, using patterns in which regularity is minimized. Finally, Experiment 3 is performed with a selection of colors on a wider variety of patterns. Given the convincing demonstrations by Fuchs, we initially hypothesize color assimilation caused by regularity. It should be clear that our main interest in color induction is the use of that phenomenon as a method to test the competition between proximity and regularity as grouping factors, not to establish the conditions to obtain contrast or assimilation, let alone their underlying peripheral or central mechanisms.

\section{Experiment 1: Regularity}

\subsection{Methods}

\subsubsection{Participants}

Eight volunteers (students, visitors and members of the Laboratory of Experimental Psychology at the University of Leuven) participated in the experiment. They were all naive with respect to the goal and design of the experiment. All participants reported to have normal acuity and color vision. 


\subsubsection{Stimuli}

All stimulus configurations were series of colored elements. First we will discuss the various manipulations on the positional arrangements of the local elements within each configuration. After that, we will discuss the manipulations on color.

2.1.2.1. Position. The stimuli were composed of linear arrangements of seven unfilled circles. In Fig. 4(a), the basic configuration is shown with the circles numbered from 1 to 7 . As will be explained in the next paragraph, the observers had to compare the color of the Test Circle (always the middle circle, number 4) with the color of the circles to its left and right. All positional arrangements were constructed by a shift to the left or to the right of the second and/or the sixth circle.

There were two stimulus subsets. In Stimulus Subset 1, the circles were arranged symmetrically around the Test Circle. The left panel in Fig. 4(b) shows the three con-

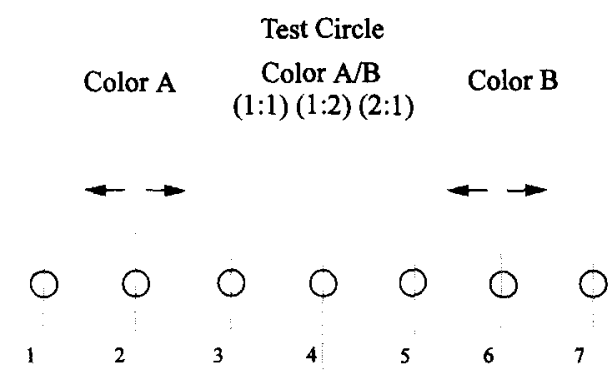

A

Subset 1

Color A Color B

C1 000000000

$\mathrm{C} 20+00000$

C3 00 O
Color A Color B

Subset 2

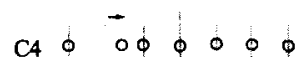

C6 0 ○

C5 00000000

\section{B}

Fig. 4. Stimulus configuration in Experiment 1. In $\mathrm{A}$, we show the basic configuration on the basis of which all other stimuli were constructed. The configurations were constructed by means of a shift of $\mathrm{Cir}$ cles 2 and 6 to the left and right (indicated by the arrows). In B, the two stimulus subsets are shown. 
figurations within this set, labelled $\mathrm{Cl}-\mathrm{C} 3$. Configuration $\mathrm{C} 1$ is the basic configuration from which Configuration $\mathrm{C} 2$ is derived by shifting Circle 2 and Circle 6 towards the Test Circle, and from which Configuration $\mathrm{C} 3$ is derived by shifting Circle 2 and Circle 6 away from the Test Circle. The shift was always half of the distance between the circles in the basic configuration. In Stimulus Subset 2, the configurations were only partly regular (see the right panel of Fig. 4(b)). Configuration C4 is derived from the Configuration $\mathrm{Cl}$ by shifting Circle 2 towards the Test Circle, whereas Configuration $\mathrm{C} 5$ requires a shift of Circle 2 away from the Test Circle. Configurations $\mathrm{C} 6$ and $\mathrm{C} 7$ are based on similar shifts, but applied to Circle 6. By means of this manipulation, the circles on one side of the Test Circle - either left or right - are spaced equidistantly (i.e., this side is regular), whereas on the other side of the Test Circle the distances between the circles are unequal (i.e., this side is irregular). To control for possible effects of orientation and to enhance the generalizibility of the experimental results, the seven patterns $(\mathrm{Cl}-\mathrm{C} 7)$ were presented in four different orientations: horizontal, vertical, and two diagonal versions (top-left to bottomright, and bottom-left to top-right); so, totally there were 28 configurations.

2.1.2.2. Color. The color of the circles on one side of the Test Circle was always different from the color of the circles on the other side of the Test Circle. Furthermore, within each trial, the circles on the left side of the Test Circle were colored the same, and the circles on the right side of the Test Circle were colored the same. The colors of these circles were selected from one of five color pairs. The colors within these pairs were drawn from the color palettes offered by Turbo Pascal 4.0 (Borland Inc.). These five color pairs were selected on the basis of a few pilot experiments in which the authors and some of their colleagues served as participants. We will refer to these color pairs by the following names: purple/red, pink/brown, red/pink, green/yellow, and blue/green. Note that these color names are rough category-names and only serve to distinguish the two colors within each color pair. Colors with the same names (but within different color pairs) are actually slightly different from each other.

In Table 1, the CIE $(x, y)$ chromaticity coordinates of the colors are given. To determine these coordinates, the colors on the color monitor used in this study were matched with adjustable colors on a BARCO color monitor linked to a PDP 11/ 23 computer (Wittebrood et al., 1981). We also present the Turbo Pascal parameters (color palette, color number) used for generating the colors. Notice, however, that the actual colors on the monitor screen do not depend uniquely on these Turbo Pascal parameters but differ when a different monitor would be used. In Table 1, also the luminances $\left(\mathrm{cd} / \mathrm{m}^{2}\right)$ are given for each color. They were measured with a Pritchard SPECTRA photometer. Notice that the colors within a pair are not isoluminant. Within a color pair, however, the relative luminance differences are rather small.

The Test Circle always had an intermediate color, and was made up of a mixture of pixels of both colors within a color pair. For each color pair (say Colors A and B), there were three possible color mixtures for the Test Circle. One mixture used a $1: 1$ pixel rate of Colors A and B. In that case, the Test Circle was made up of half of the pixels of Color A and half of the pixels of Color B. The other two mixtures had pixel 
Table 1

Specification of the colors used in the experiments. For each color, the CIE $(x, y)$ chromaticity coordinates, the luminances $\left(\mathrm{cd} / \mathrm{m}^{2}\right.$ ), and the Turbo Pascal (4.0 Borland Inc.) parameters (color palette and color number) are given. Notice, however, that the actual colors on the monitor screen do not depend uniquely on these parameters but are monitor-dependent

\begin{tabular}{|c|c|c|c|c|c|}
\hline \multirow[t]{2}{*}{ Color } & \multicolumn{2}{|c|}{$\operatorname{CIE}(x, y)$} & \multirow[t]{2}{*}{$\mathrm{L}\left(\mathrm{cd} / \mathrm{m}^{2}\right)$} & \multicolumn{2}{|c|}{ Pascal parameters } \\
\hline & $X$ & $Y$ & & 'Palette' & 'Number' \\
\hline \multicolumn{6}{|c|}{ Color pair 1} \\
\hline Purple & 0.427 & 0.225 & 4.8 & $(1,12)$ & 1 \\
\hline Red & 0.594 & 0.322 & 4.3 & $(1,12)$ & 4 \\
\hline \multicolumn{6}{|c|}{ Color pair 2} \\
\hline Pink & 0.391 & 0.305 & 8.9 & $(1,28)$ & 1 \\
\hline Brown & 0.497 & 0.403 & 8.4 & $(1,28)$ & 6 \\
\hline \multicolumn{6}{|c|}{ Color pair 3} \\
\hline Red & 0.609 & 0.329 & 11.1 & $(1,36)$ & 1 \\
\hline Pink & 0.498 & 0.319 & 15.6 & $(1,36)$ & 12 \\
\hline \multicolumn{6}{|c|}{ Color pair 4} \\
\hline Green & 0.314 & 0.519 & 47.7 & $(1,1)$ & 10 \\
\hline Yellow & 0.405 & 0.477 & 57.3 & $(1,1)$ & 14 \\
\hline \multicolumn{6}{|c|}{ Color pair 5} \\
\hline Blue & 0.233 & 0.283 & 23.0 & $(1,3)$ & 1 \\
\hline Green & 0.327 & 0.548 & 20.1 & $(1,3)$ & 2 \\
\hline
\end{tabular}

rates of $1: 2$ and $2: 1$. Each circle was in fact made up of twelve pixels. In case the pixel rate was $1: 1$, two version of the Test Circle were possible; in either case, there were six pixels with color A and six pixels with color B (see Fig. 5(a)). In the experiment, the Test Circle for each trial with a $1: 1$ pixel rate was randomly selected from these two possibilities. In case the pixel rate of Color A and B was $2: 1$ or $1: 2$ (based on actual pixel rates of $8: 4$ and $4: 8$, respectively), the Test Circle was composed as indicated in Fig. 5(b).

For each color pair (say A and B), the colors could be assigned to a configuration in two different ways. First, Color A could be assigned to the circles on the left side of the Test Circle and Color B to the right side of the Test Circle. The second possibility was the reverse. Together with the three intermediate colors of the Test Circle, there are six different color settings per color pair.

2.1.2.3. Number of trials. Each participant saw 840 stimuli: Number of configurations (7) $\times$ Number of orientations $(4) \times$ Number of color pairs $(5) \times$ Number of color settings $(6)=840$.

\subsubsection{Apparatus}

The experiment was carried out on an IBM-compatible computer with a 486 INTEL processor. The stimuli were displayed on a color monitor (SMILE, Taiwan, ROC) with a refresh rate of $60 \mathrm{~Hz}$ and a $640 \times 480$ pixel display area. 


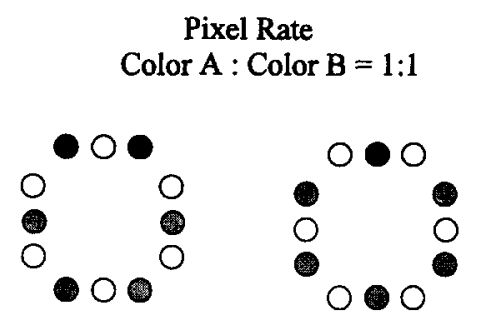

A
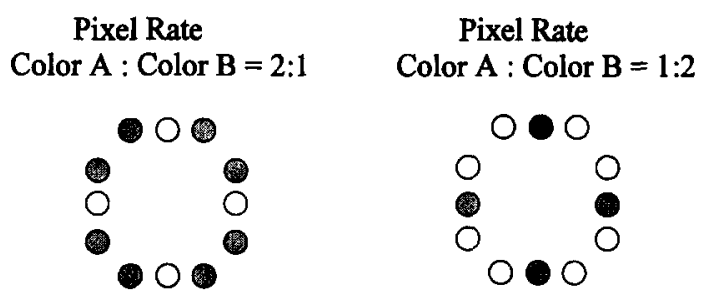

B

Fig. 5. Each circle of the stimuli in Fig. 4 was made up of 12 pixels. In A, the two possible pixel settings for the pixel rate of $1: 1$ are shown. In $B$, the pixel settings for the pixel rates $2: 1$ and $1: 2$ are shown.

\subsubsection{Procedure}

In Fig. 6, the sequence of events and spatial lay-out in one trial is shown. Each trial started with a black screen for $1000 \mathrm{~ms}$ followed by a fixation cross in the middle of the monitor screen. After $500 \mathrm{~ms}$, the fixation cross disappeared and after $17 \mathrm{~ms}$ (due to screen refreshment), the stimulus configuration appeared on the screen. The Test Circle appeared at exactly the same position as the fixation cross. The stimulus configuration remained on the screen until a response was given by one of two

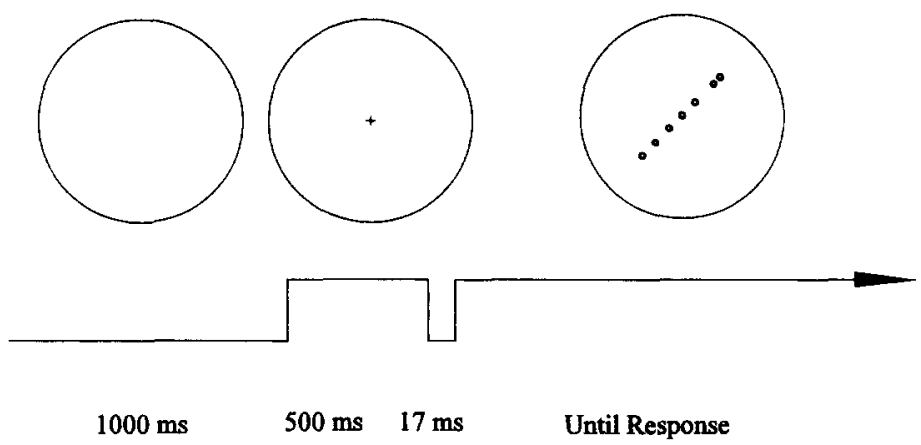

Fig. 6. The sequence of events within an experimental trial: First a black screen $(1000 \mathrm{~ms})$, then a fixation cross $(500 \mathrm{~ms})$, and after $17 \mathrm{~ms}$ the stimulus configuration appeared until a response was given. 
buttons. The participants were instructed to press the left button if the color of the Test Circle was more similar to the circles left of the Test Circle. Correspondingly, the right button had to be pressed if the color of the Test Circle was more similar to the circles right of the Test Circle. When the configuration was presented vertically, the left button had to pressed if the color of the Test Circle was more similar to the circles above the Test Circle, and the right button had to be pressed if the color of the Test Circle was judged to be more similar to the circles below the Test Circle.

The diameter of the circles on the monitor screen was $2 \mathrm{~mm}$, whereas the length of the whole configuration was approximately $104 \mathrm{~mm}$. The visual angle of the whole configuration subtended less than $5^{\circ}$. Each subject received 840 stimuli, in complete random order. The duration of the total experiment was approximately $50 \mathrm{~min}$. After each 50 trials, there was a pause. The duration of the pause could be determined by the participants. Before running the experiment, the participants practised on a set of 50 stimuli, randomly selected from the 840 experimental stimuli.

\subsubsection{Hypothesis}

We hypothesize that the Test Circle is perceptually grouped with the equidistantially spaced circles (i.e., the regular segment). Considering the early findings of Fuchs (1923), we further hypothesize that color similarity judgements of the Test Circle tend to the color of the elements of the regular segment in each configuration. Thus, we expect that, on the whole, the color of the test circle is most frequently judged to be most similar with the color of the elements in the regular segment.

\subsection{Results and discussion}

\subsubsection{Global results}

We first consider the global results on all stimuli. In Table 2, the total number of responses, across all pixel rates, are given for each color pair on both stimulus subsets. A possible dominance of either color A or color B within a color pair can easily be determined by inspecting Stimulus Subset 1, as for those configurations there is no positional difference between the local elements on either side of the Test Circle. For example, in Table 2 it can be seen that within Stimulus Subset 1, the Test Circle is rated to look more purple 163 times, whereas it looks more reddish 413 times. Within Stimulus Subset 2, not only the colors between left and right differ but also the positions of the local elements. Remember that for a specific stimulus, there were two possibilities. The color of the Test Circle had to be compared with either "color A in a regular segment" and "color B in an irregular segment" or with "color B in a regular segment" and "color A in an irregular segment". So, for example, in one stimulus the color of the elements of the regular segment was purple and the color of the elements of the irregular segment was red. In a second stimulus, the color of the elements of the regular segment was red and the color of the elements in the irregular segment was purple. Considering the responses within the red/purple color pair, the color of the Test Circle was judged to look more purple 92 times when the irregular segment of the stimulus was purple, whereas 151 times the same judgement was made when the regular segment was purple. 
Table 2

Results from Experiment 1. The total number of responses, across all pixel rates, are given for each color pair on both stimulus subsets. With respect to Stimulus Set 2, a further distinction is made between Irregular Segment and Regular Segment

\begin{tabular}{llcl}
\hline Color & Stimulus subset 1 & \multicolumn{2}{c}{ Stimulus subset 2 } \\
\cline { 4 - 4 } & & Irregular segment & Regular segment \\
\hline Color pair 1 & 163 & 92 & 151 \\
Purple & 413 & 233 & 292 \\
Red & & & \\
Color pair 2 & 300 & 176 & 233 \\
Pink & 276 & 151 & 208 \\
Brown & & & \\
Color pair 3 & 346 & 220 & 252 \\
Red & 230 & 132 & 164 \\
Pink & & & \\
Color pair 4 & 134 & 69 & 143 \\
Green & 442 & 241 & 315 \\
Yellow & & & 304 \\
Color pair 5 & 418 & 237 & 147 \\
Blue & 158 & 80 & \\
Green & & & \\
\hline
\end{tabular}

The numbers of both stimulus subsets cannot be compared directly with each other. Stimulus Subset 1 contains 576 responses for each color pair, whereas Stimulus Subset 2 contains 384 responses for each of two above-mentioned possibilities per color pair. That is, for each color pair (Color A/Regularity versus Color B/Irregularity; Color B/Regularity versus Color A/Irregularity) the observed responses sum up to a total of 384 responses. For a direct comparison between the numbers in Table 2, we computed the corresponding percentages of preference for each color and for each of three relative positional properties (irregularity, ambiguity, and regularity).

These percentages are depicted in Fig. 7. In the upper part, the bars for the dominant color, that is the color within a color pair which is chosen most frequently, are plotted as a function of the relative positional property. In the lower part, the bars for the nondominant colors are plotted. Inspecting the graph, it can be seen that, in general, the color of the Test Circle is most frequently judged to be more similar to the color of the circles in the regular segment, indicating color assimilation induced by regularity. Notice that the bars from the dominant colors and the nondominant colors are complementary with respect to each other. Recall that for each configuration there is a version in which the dominant color is assigned to one segment (e.g., the regular segment) and the nondominant color to the other segment (e.g., the irregular segment), and vice versa. The percentages of preference for a specific color within each color pair, however, are not complementary as for each subject these percentages in fact stem from different stimuli. Therefore, in the analysis, we consider percentages of preference for segments of a single color, for example, the dominant color. 
Preference (\%)

Dominant colors

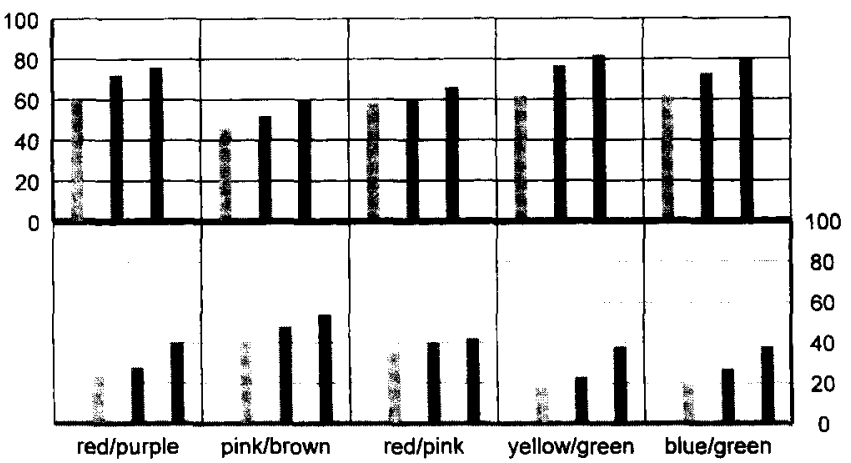

Relative positional property of preferred segment

Irregularity

ambiguity

Regularity

Preference (\%)

Non-dominant colors

Fig. 7. Results from Experiment 1. The graph depicts the percentages of preference for each color and for each of three positional properties (irregularity, ambiguity, regularity). In the upper part, the bars for the dominant colors are plotted. In the lower part, the bars for the nondominant colors are plotted. It can be seen that, for all colors, the color of the Test Circle is judged to be more similar to a specific color if that specific color is used in the regular segment of the stimulus configurations.

Inspecting the graphs of Fig. 7, it can be noticed that all trends are in the expected direction. The Friedman ANOVA By Ranks on the overall percentages is significant: $\left.X^{2}(2, N=8)=10.00, p<0.01\right)$. The Friedman ANOVA By Ranks for each color pair on the mean percentages of preference calculated per participant, yields the following results. Color Pair 1 (purple/red): $X^{2}(2, N=8)=12.25, p<0.01$. Color Pair 2 (pink/brown): $X^{2}(2, N=8)=6.25, p<0.05$. Color Pair 3 (red/pink): $X^{2}(2$, $N=8)=3.20$ (n.s.). Color Pair 4 (green/yellow): $X^{2}(2, N=8)=4.06$ (n.s.). Color Pair 5 (blue/green): $X^{2}(2, N=8)=12.97, p<0.01$. So, for three color pairs $(1,2$, and 5), there appears to be a significant color assimilation effect induced by regularity; for the remaining two pairs, the trends are similar but not statistically reliable.

Notice that the two color pairs which yielded no significant assimilation effect (Color Pairs 3 and 4) have the largest difference in the luminances between the two colors within the color pair (see Table 1). Perhaps this greater difference in luminance might have weakened the assimilation effect. Such a possible effect of luminance would fit with observations that have been reported by Agostini and Proffitt (1993), who showed that perceptual grouping of dot patches having different luminances might even induce contrast effects. In their study, however, the luminance differences were much greater than the maximum difference applied here, while the stimuli were achromatic.

\subsubsection{Pixel rate}

We now focus on Stimulus Subset 2 in which the positions of elements on both sides of the Test Circle differ from each other and take a closer look at the effects of pixel rate on the preferences. The usage of different pixel rates leads us to the following classifications. If the pixel rate is $1: 1$, we will classify the mixture as $B a l$ - 
anced. If the pixel rate is $1: 2$ or $2: 1$, two levels have to be distinguished: If twothirds of the Test Circle consist of the same color as the regular segment, the mixture is said to be Congruent as it supports the preference for the color in the regular segment. Similarly, if only one-third of the Test Circle consists of the same color as the regular segment, the color mixture is said to be Incongruent. Notice that the latter classifications are indeed meaningful only for the configurations of Subset 2 in which one segment is always regular, whereas the other is not.

In Fig. 8, the percentage of preference for the color of the regular segment has been plotted as a function of this classification. It can be observed that the percentage of preference for the regular segment gradually decreases going from Congruent to Balanced to Incongruent. The Friedman ANOVA By Ranks on the overall differences between the mean preferences per subject on the three classes yields: $X^{2}(2$, $N=8)=14.25, p<0.01$. Obviously, if there was no color assimilation effect, one also would have expected the decrease in percentage of preference for the regular segment, or negative "slope", going from Congruent to Balanced to Incongruent. However, in that case the percentage of preference for the color of the regular segment would have been centred around the 50\% level for the Balanced color mixture. (Notice that the experimental design is completely balanced with respect to both positions and colors.) The mean values being above the 50\% level indicate the color assimilation effect and, in fact, were examined already with respect to Fig. 7. Furthermore, it can be noticed in Fig. 8 that the color pairs for which significant assimilation effects were obtained (Color Pairs 1, 2, and 5, see Section 2.2.1.) have the most moderate "slopes", that is, they have the smallest difference between the preferences for the regular segment on the Congruent condition and the Incongruent condition. Obviously, the smaller the difference between the preferences for the regular segment on the Congruent condition and the Incongruent condition the lesser the physical color setting is decisive in the color judgements.

Preference for color regular segment (\%)

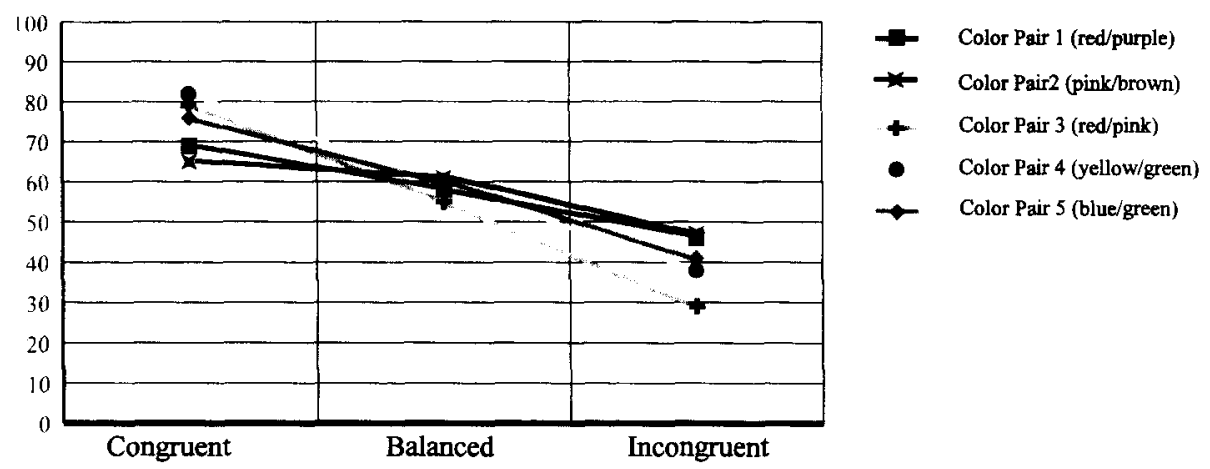

Fig. 8. Results from Experiment 1. The percentage of preference for the color of the regular segment has been plotted for the three classes Congruent, Balanced and Incongruent. Congruent: Two-third of the Test Circle consisted of the same color as the regular segment. Balanced: The Test Circle had a pixel rate of 1 : 1. Incongruent: One-third of the Test Circle consisted of the same color as the regular segment. 


\subsubsection{Orientation}

We further analyzed whether there was an effect of the orientation of the stimulus on the monitor screen. We restricted the analyses to Color Pairs 1, 2, and 5 because for these color pairs significant results on the effect of regularity were obtained. As mentioned, the stimuli were displayed in four different orientations on the monitor screen. The percentages of judgements for which the color of the Test Circle was judged to be more similar to the color of the regular segment for each of four orientations was as follows: horizontal: $61.8 \%$, vertical: $54.7 \%$, top-left to bottom-right: $58.3 \%$, and bottom-left to top-right: $56.9 \%$. The Friedman ANOVA By Ranks yields: $X^{2}(3, N=24)=7.85, p<0.01$. Most noticeable is the difference between the percentages on the horizontal and the vertical orientation. The higher preference for the regular segments in the horizontal orientation is in agreement with findings that indicate the higher susceptibility of the visual system for regularities in horizontal orientations (e.g., Wagemans, 1995). We must be cautious, however, in deducing such a differential susceptibility based on the present data as there might have been some response interference caused by the differential correspondence between the orientation of the stimulus on the monitor screen and the orientation of the response buttons. The orientation of these buttons corresponded best with the segments in the horizontal orientation and worst with the vertical orientation which could have affected the number of response mistakes correspondingly.

\subsubsection{Relative proximity}

Finally, we have analyzed whether there was an effect of the direction of the shifted circle in the irregular segment of the series in Stimulus Subset 2. The analysis was again performed on Color Pairs 1, 2, and 5. Considering the subset of all stimuli for which irregularity was induced by a shift of the second or sixth circle towards the Test Circle (i.e., Configurations C4 and C6), the color of the Test Circle was judged to be most similar to the circles of the regular segment in $58.7 \%$ of all cases. For the subset of all stimuli for which irregularity was induced by a shift of the second or sixth circle away from the Test Circle (i.e., Configurations C5 and C7), the color of the Test Circle was judged to be most similar to the circles of the regular segment in $57.1 \%$ of all cases. According to the Wilcoxon Matched Pairs Analyses By Ranks on the mean preferences per participant for Color Pairs 1, 2, and 5, this difference is not significant: $z=0.543$ (n.s.). From this result it can be deduced that, for the present configurations, the relative proximity is not a relevant factor with respect to the assimilation effects.

\section{Experiment 2: Proximity}

In the previous experiment, we have demonstrated that color assimilation can be induced by regularity, that is, the color of an element tends to look more like the color of a group of elements with which that element constitutes a regular configuration than is the case with an irregular group of elements. To compare assimilation between regular and irregular groups of elements, the positions of the elements in 
the groups inevitably differ from each other. In Experiment 1, this difference resulted from a shift of one element in the basic configuration. This shift was either towards or away from the Test Circle. The results on the differences between these two kinds of stimuli on the preferences for the regular segment do not suggest an influence of position. Obviously, from this result we cannot deduce that there is no effect of distance on color assimilation. For example, the differences in the effects of position might be much stronger if there is no regular group at all. Furthermore, in Experiment 1 , there was always another element between the shifted element and the Test Circle. In Experiment 2, effects of proximity on color judgements are examined more directly.

\subsection{Methods}

\subsubsection{Participants}

Eight new volunteers from the same pool as in Experiment 1 participated in the experiment. They were all naive with respect to the goal and design of the experiment and reported to have normal acuity and color vision.

\subsubsection{Stimuli}

3.1.2.1. Position. Within each stimulus, the local elements were the same as in the previous experiment. This time, however, each stimulus consisted of only three circles. In Fig. 9(a), the basic configuration is shown. Again, we make a distinction between two stimulus subsets. Stimulus Subset 1 consists of just one configuration, namely the basic configuration, labelled $\mathrm{Cl}$ in Fig. 9(b). The four other configurations were created by four (different) shifts of the Test Circle. Notice that, in contrast to Experiment 1, the Test Circle was shifted. This was done to preserve (as in Experiment 1) the same visual angle for all the stimuli. Moreover, as in Experiment 1, the Test Circle was positioned in the centre of the screen. One configuration is constructed by means of a shift of the Test Circle to the left, such that the distance between the left circle and the Test Circle was two-third of the initial distance, labelled $\mathrm{C} 2$ in Fig. 9(b). The second configuration has also been made by a shift to the left, but this time resulting in a distance between the left circle and the Test Circle of one-third of its initial distance, labelled C3 in Fig. 9(b). The other two configurations have similar shifts of the Test Circle to the right, labelled C4 and C5 in Fig. 9(b).

3.1.2.2. Color. The manipulations with respect to color were the same as in the first experiment, that is, the same five color pairs were used, with the same three possible pixel rates for the color of the Test Circle $(1: 1 ; 2: 1$; and $1: 2)$. Furthermore, for each configuration, and for each color pair, the color assignments of the two colors to the left and right side of the Test Circle were balanced.

3.1.2.3. Number of trials. As mentioned above, there were five different positional configurations. To collect the same number of data points as in Experiment 1 on 


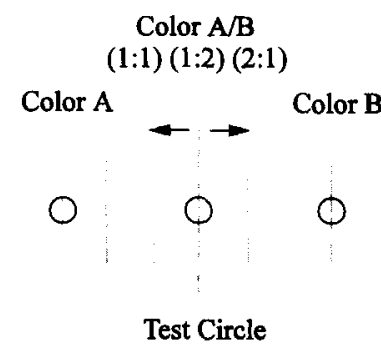

A

Subset 1

Color A Color B

$\mathrm{Cl}$

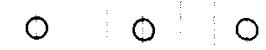

Subset 2

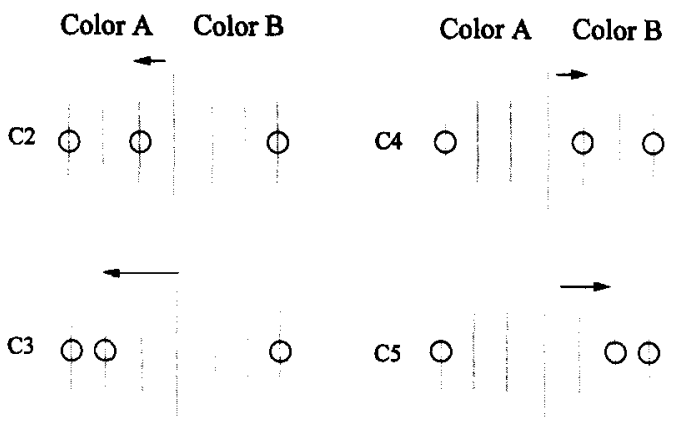

\section{B}

Fig. 9. Stimulus configuration used in Experiment 2. In A, the basic configuration is shown. The configurations were constructed by means of different shifts of the central circle to the left and right (indicated by arrows). In B, the two stimulus subsets are shown.

the stimuli that were ambiguous with respect to position, Configuration $\mathrm{Cl}$ was presented three times to each subject. The total amount of stimuli that each subject received was Number of configurations $(3+4=7) \times$ Number of orientations $(4) \times$ Number of color pairs $(5) \times$ Number of color settings $(6)=840$.

\subsubsection{Procedure}

The experimental procedure was the same as in Experiment 1. The stimuli were presented such that the fixation cross and the Test Circle always appeared centrally.

\subsubsection{Hypothesis}

We hypothesize that the Test Circle is perceptually grouped with the most proximate circle. We expect that, on the whole, the color of the test circle is most frequently judged to be most similar to the color of the most proximate element. 


\subsection{Results and discussion}

\subsubsection{Global results}

First, we consider the global results on all stimuli. In Table 3 , the total number of responses, across all pixel rates, are given for each color pair on both stimulus subsets. The corresponding percentages, computed in the same way as in Experiment 1, are depicted in Fig. 10. A brief inspection of Fig. 10 reveals that there is much more variation between the different color pairs and that the effects of position on color appearance are not so uni-directional as in Experiment 1. The Friedman ANOVA By Ranks on the overall percentages was not significant: ANOVA $X^{2}(2$, $N=5$ ) $=0.40$ (n.s.). The Friedman ANOVA By Ranks for each color pair on the mean percentage of preference calculated for each participant, yields the following results. Color Pair 1 (purple/red): $X^{2}(2, N=8)=7.75, p<0.05$; Color Pair 2 (pink/brown): $X^{2}(2, N=8)=12.25, p<0.01$; Color Pair 3 (red/pink): $X^{2}(2$, $N=8)=2.06$ (n.s.); Color Pair 4 (green/yellow): $X^{2}(2, N=8)=5.25$ (n.s.); Color Pair 5 (blue/green): $X^{2}(2, N=8)=3.16$ (n.s.). Notice that two of the three color pairs that showed a significant assimilation effect induced by regularity in Experiment 1, also show a significant assimilation effect induced by proximity. These are Color Pair 1 (purple/red) and Color Pair 2 (pink/brown). Again, there is a correspondence between the differences in luminance of the colors within color pairs and the assimilation effects. Within the context of our hypothesis, the best results are obtained for the

Table 3

Results from Experiment 2. The total number of responses, across all pixel rates, are given for each color pair on both stimulus subsets. With respect to Stimulus Set 2, a further distinction is made between $E x$ treme Element and Proximate Element

\begin{tabular}{llll}
\hline Color & Stimulus subset 1 & Stimulus subset 2 & \\
\cline { 4 - 4 } & & Extreme element & Proximate element \\
\hline Color pair 1 & 133 & 72 & 142 \\
Purple & 443 & 242 & 312 \\
Red & & & \\
Color pair 2 & 288 & 150 & 257 \\
Pink & 288 & 127 & 234 \\
Brown & & & \\
Color pair 3 & 370 & 238 & 231 \\
Red & 206 & 153 & 146 \\
Pink & & & \\
Color pair 4 & 155 & 153 & 231 \\
Green & 421 & 284 & \\
Yellow & & & 286 \\
Color pair 5 & 371 & 248 & 136 \\
Blue & 205 & 98 & \\
Green & & & \\
\hline
\end{tabular}


Preference (\%)

Dominant colors

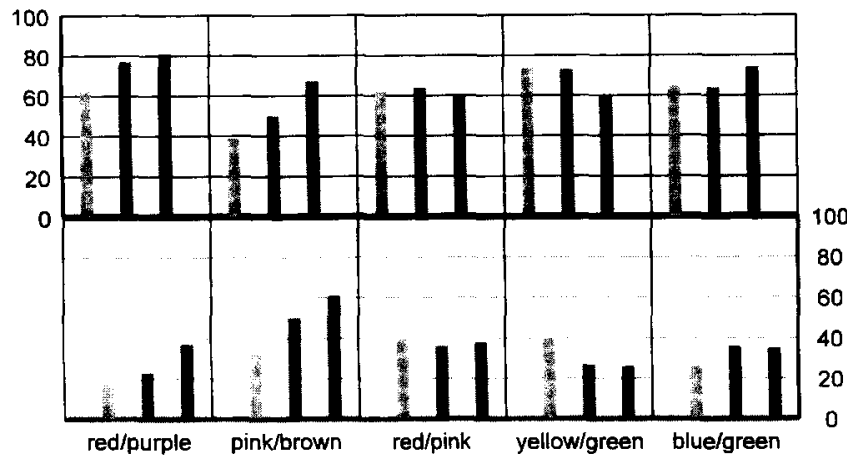

Positional property of preferred element

Extremity

Ambiguity

- Proximity

Preference (\%)

Non-dominant colors

Fig. 10. Results from Experiment 2. The graph depicts the percentages of preference for each color and for each of three positional properties (extremity, ambiguity, proximity). It can be seen that, for Color Pairs 1 , 2, and 5, the color of the Test Circle is judged to be more similar to a specific color if that specific color is equal to the color of the proximate element. This is not so for Color Pairs 3 and 4.

two color pairs with the smallest difference in luminance (i.e., Color Pairs 1 and 2; see Table 1), whereas almost opposite tendencies were observed for the two color pairs with the largest difference in luminance (i.e., Color Pairs 3 and 4; see Table 1).

\subsubsection{Pixel rate}

We now focus on Stimulus Subset 2, taking a closer look at the effect of pixel rate. In Fig. 11, the percentage of judgements of color similarity between the Test Circle

Preference for color proximate element (\%)
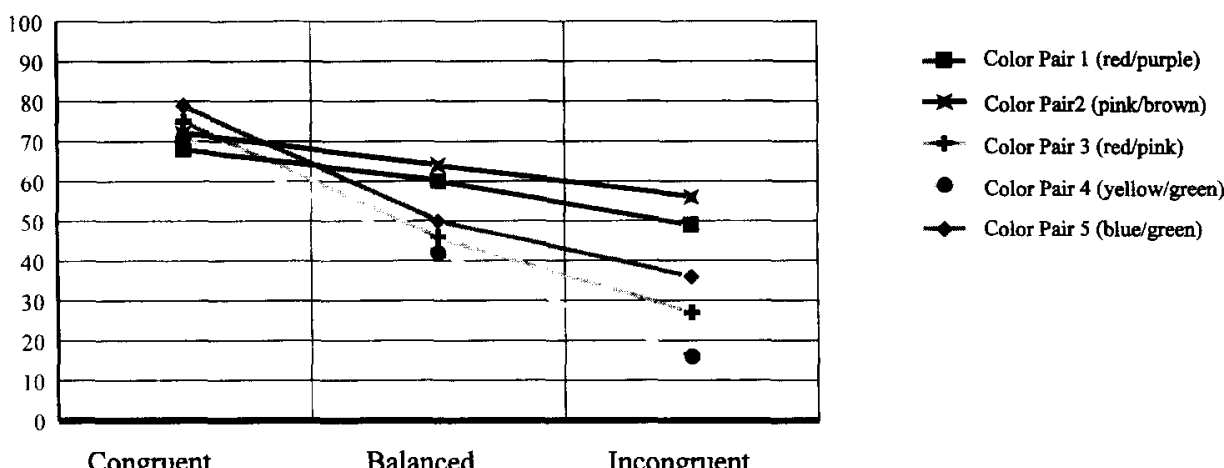

Fig. 11. Results from Experiment 2. The percentage of preference for the color of the regular segment has been plotted for the three classes Congruent, Balanced and Incongruent. 
and the most proximate circle has been plotted analogously to Fig. 8 for the three color mixture classes: Congruent, Balanced, and Incongruent. This time, the Congruent mixture indicates that the Test Circle contains twice as many pixels from the color of the most proximate circle as from the color of the most extreme circle. Accordingly, the Incongruent mixture indicates that the Test Circle contains half as many pixels from the color of the most proximate circle as from the color of the most extreme circle. Like in Experiment 1, a quasi-linear function between the percentage of preference and the three classes can be observed. A Friedman ANOVA By Ranks on the overall differences between the preferences on these three subsets yields: $X^{2}(2$, $N=8)=14.25, p<0.01$.

In Fig. 11, the much larger variation in the data, in comparison with Experiment 1, can be observed clearly. Notice that in the Balanced color mixture condition, the percentages for Color Pairs 1 and 2 are clearly above the $50 \%$ level, whereas they are not so for the other color pairs. These results indicate that proximity may be more likely to induce contrast effects than regularity does. Like in Experiment 1, the color pairs with the smallest difference between the results in the Congruent condition and the Incongruent condition, or say the most moderate "slope", are most susceptible to the color assimilation effect. As the slope can be considered as an indication for the similarity of the colors, these results show that assimilation effects occur if the perceptual distance between the colors within a color pair are within certain limits. Notice, however, that from the present experiments, we cannot deduce what the exact properties of an ideal color pair are. Such an examination lies beyond the scope of the present paper. The aspect that is of interest here is that, given our experimental set-up, color pairs do exist for which both regularity and proximity induce color assimilation.

\subsubsection{Orientation}

Analogously to Experiment 1, we have analyzed whether there was an effect of the orientation of the stimulus on the monitor screen. This time, we restrict the analyses to Color Pairs 1 and 2, as for these color pairs a significant overall effect of proximity on color assimilation was obtained. The percentages of judgements for which the color of the Test Circle was judged to be more similar to the color of the most proximate circle for each of four orientations were as follows: horizontal: $66.1 \%$, vertical: $59.4 \%$, top-left to bottom-right: $61.5 \%$, and bottom-left to top-right: $59.1 \%$. The Friedman ANOVA By Ranks yields: $X^{2}(3, N=16)=6.66$ (n.s.). Analogously to the results on Experiment 1, the percentages tend to be the highest for horizontal orientations. A rather speculative explanation for the higher preference for the most proximate element in the horizontal orientation (although not statistically significant) would be that horizontal distances are likely to be perceived shorter than vertical distances (i.e., the so-called horizontal-vertical illusion). This would imply that assimilation by proximity is mediated by perceptual processes after the illusion has taken place. Although we do not exclude this possibility, the differences in responses might, similar to Experiment 1, have been caused by the differential correspondence between the orientation of the stimulus on the monitor screen and the orientation of the response buttons. 


\subsubsection{Degree of proximity}

Finally, the relevance of assimilation due to proximity has been examined by analyzing the influence of the degree of proximity on the color judgements. As explained in the stimulus construction, there were two degrees of proximity (see Fig. 9). Considering the subset of all stimuli which were constructed by means of the largest shift of the Test Circle to the left or to the right, the color of the Test Circle was judged to be most similar to the proximate circle in $66.8 \%$ of all cases. Considering the subset of all stimuli for which the Test Circle has made the smallest shift, the color of the Test Circle was judged to be most similar to the proximate circle in $56.3 \%$ of all cases. According to the Wilcoxon Matched Pairs Analyses By Ranks on the mean preferences per participant for Color Pairs 1 and 2, there is a significant effect of the degree of proximity and the color assimilation effect: $z=3.41, p<0.001$.

\section{Experiment 3: Regularity versus proximity}

In Experiments 1 and 2, it has been demonstrated that for specific color pairs and their mixtures both regularity and proximity induce color assimilation. In Experiment 3 , these color assimilation effects are examined on a broader range of positional variations of elements within a configuration. By doing so, competitive effects of proximity and regularity can be considered as well.

\subsection{Methods}

\subsubsection{Participants}

Eight new volunteers from the same pool as in the previous experiments participated in the experiment. They were all naive with respect to the goal and design of the experiment and reported to have normal acuity and color vision.

\subsubsection{Stimuli}

4.1.2.1. Position. In the stimulus construction, we have made use of the same basic configuration as in Experiment 1. This time, however, more positions of the circles have been varied. The stimulus construction is based on systematic shifts of circles within the basic configuration. In Fig. 12(a), the basic configuration is shown with all possible shifts of the circles indicated by arrows. Except for the basic configuration, each experimental configuration is a result of a shift of one or two circles, one on each side of the Test Circle. The shifts were performed on Circles 2, 3, 5, or 6. In the following, we will refer to the circles closest to the Test Circle (i.e., Circles 3 and 5 ) with the Roman number I, and to Circles 2 and 6, with the Roman number II. Three subsets of stimuli can be distinguished.

The circles within the configurations of Stimulus Subset 1 are all symmetrically arranged around the Test Circle, resulting from shifts of Circle I or Circle II in opposite directions. The symmetrical basic configuration is considered to be a member 


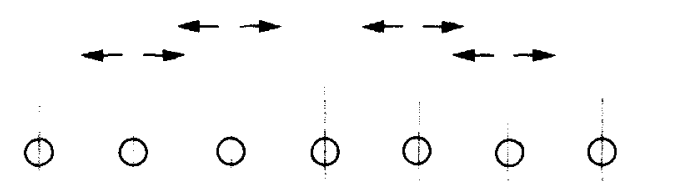
Circle-II Test Circle Circle-II
Circle-I Circle-I

A

Subset 1

C1

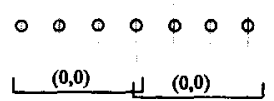

$\mathrm{C} 2$

$\circ \circ$ O०० 0 o

$+(0,0)+(0,0)$

C3 0 0. 0 ००००

$(0,0) \lcm{(0,0)}$

0.00000

$\mathrm{C} 4$

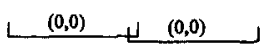

C5

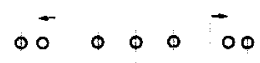

$(0,0)+1 \quad(0,0)$
Subset 2

Subset 3

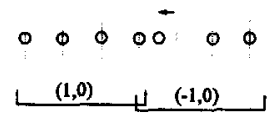

C7

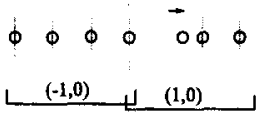

$00 \phi 000^{-}$

C8

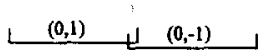

C9

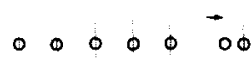

L $(0,-1) \perp \quad(0,1)$

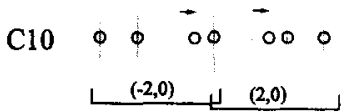

C1

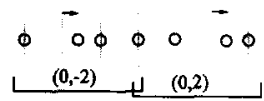

$\mathrm{C} 12$

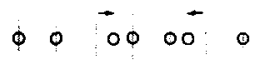

لـ

C13
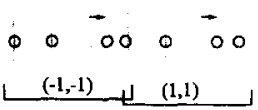

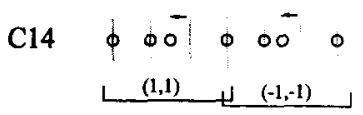

C15

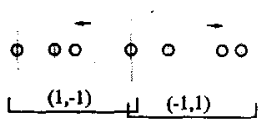

B

Fig. 12. Stimulus configuration used in Experiment 3. In A, the basic configuration, similar to the one of Experiment 1, is shown. The configurations were constructed by means of shifts with Circle I and Circle II as indicated by the arrows. In B, the three stimulus subsets are shown. The numbers below the segments refer to the relative position of Circle I and Circle II in the two stimulus segments and are referred to as the Dprox coordinates (see text for more details). 
of Stimulus Subset 1 as well. In Fig. 12(b), these configurations have been labelled C1-C5. In Stimulus Subsets 2 and 3, the spacing between the local elements on the left side of the Test Circle was different from the spacing on the right side of the Test Circle. The construction of Stimulus Subset 2 proceeded by a shift of only one circle within the basic configuration to the left or to the right. As a result, one side of the configuration is always equidistantly spaced, whereas the other half is not. These configurations are labelled $\mathrm{C} 6-\mathrm{C} 9$. Notice that there is a mirror version for each configuration. So, there are in fact eight configurations, each label representing two versions. The construction of the configurations within Stimulus Subset 3 , proceeded by a shift of two circles, one on each side of the Test Circle. As a result, both sides are irregularly spaced. In Fig. 12(b), these six configurations are labelled $\mathrm{C} 10-\mathrm{Cl}$. There is again a mirror version for each depicted configuration.

By means of the shifts with Circles I and Circles II, the proximity of the circles with respect to the Test Circle has several degrees. To examine the joint influence of Circle I and Circle II at both sides of the Test Circle, we have determined the relative positional difference for these circles. These positional differences will be expressed in terms of the so-called Dprox values, indicated by the numbers below each of two configuration segments in Fig. 12. Each segment has two such values. The Dprox I value refers to the relative positional difference of Circle I on both sides of the Test Circle. In a similar way, the Dprox II value refers to the relative positional difference of Circle II on both sides of the Test Circle. They are calculated as follows. By convention, the distance between two neighboring circles in the basic configuration is set to a value of 2 . Now, consider a segment in which Circle I is shifted towards the Test Circle like in Configuration C6. As such a shift always is half the initial distance between Circle I and the Test Circle, the resulting distance between Circle I and the Test Circle has a value of 1 . Because Circle I on the opposing side of the Test Circle in Configuration C6 has not been shifted, the Dprox I value for that segment equals $1-2=-1$. The calculations with respect to Circle II proceed in a similar fashion. As a result, the Dprox coordinates of the right segment (as shown in Fig. 12) in Configuration $\mathrm{C} 1$ are $(-1,0)$, the first number referring to the Dprox I value and the second number to Dprox II. Obviously, the Dprox I and Dprox II values of the segments on either sides of the Test Circle always have opposing values.

4.1.2.2. Color. Only Color Pair 1 (red/purple) and Color Pair 2 (pink/brown) of the previous experiments were employed, as they evoked significant assimilation effects induced by both regularity and proximity. The manipulations with respect to the pixel rate of the Test Circle were exactly the same as in the Experiments 1 and 2.

4.1.2.3. Number of trials. Each subject received 25 configurations (the 15 configurations as shown in Fig. 12(b) plus the 10 mirror versions for the configurations within Stimulus Subsets 2 and 3), in four different orientations (horizontal, vertical, top-left to bottom-right, and bottom-left to top-right), with two color pairs, and six color settings (left-right balance $\times$ pixel rates), yielding a total of $25 \times 4 \times 2 \times 6=1200$ trials. 


\subsubsection{Procedure}

The experimental procedure was the same as in Experiments 1 and 2.

\subsubsection{Hypothesis}

The hypothesis is not as straightforward as in the previous experiments. Because of the larger variation in the positions of the circles with respect to the Test Circle, the influence of proximity on color assimilation can be examined in greater detail. We expect that, in general, the assimilation effects caused by the position of Circle I are greater than the effects caused by the position of Circle II. The preferences on Stimulus Subset 1 are again expected to take an intermediate position. Note, further, that Configurations $\mathrm{C} 8$ and $\mathrm{C} 9$, constructed by means of a shift of Circle II, have been used in Experiment 1 as well. Because Experiment 1 yielded a higher preference for the regular segment for both configurations, we expect a similar result in the present experiment. On the basis of the results of Experiment 2, however, we would expect that the assimilation due to proximity also influences the responses on Configurations $\mathrm{C} 6$ and $\mathrm{C}$, revealing a competition between regularity and proximity in the induction of color assimilation.

\subsection{Results and discussion}

\subsubsection{Global results}

Considering the whole data set and all positional differences, it appears that within the red/purple color pair, there were $30.5 \%$ judgements made for the color purple and $69.5 \%$ judgements for the color red. Within the brown/pink color pair, there were $33.3 \%$ judgements made for the color pink and $66.7 \%$ judgements for the color brown. Because of the complementarity of the color judgements, we restrict the data analysis and presentation to one color within each color pair. In the following, the preferences for the dominant colors will be presented (i.e., the colors red and brown). Obviously, analyses on absolute differences between the color judgements for any other combination of two colors from both color pairs would lead to exactly the same conclusions.

To examine the positional influence of the local elements on the color judgement, we first follow the classification made in the above paragraph on the stimulus construction. According to that classification, each segment (on either side of the Test Circle) can be rated with a Dprox I value, depending on the relative positions of Circle I on both sides of the Test Circle, and a Dprox II value, depending on the relative positions of Circle II on both sides of the Test Circle. Considering all possible segments, the values of both Dprox I and Dprox II range from -2 to 2. As explained above, a negative value of, for example, Dprox I for a given segment in a stimulus indicates that Circle I for that segment is closer to the Test Circle than Circle I in the opposing segment. In Fig. 13, the mean percentages for all possible combinations of the Dprox I values and the Dprox II values are given. For example, the height of the bar with Dprox coordinates $(-2,0)$ reflects the percentage of judgements made for the corresponding segment in Configuration $\mathrm{C} 10$ (the left segment in the configuration as shown in Fig. 12, and, of course, the right segment in the mirror version 


\section{Preference (\%)}

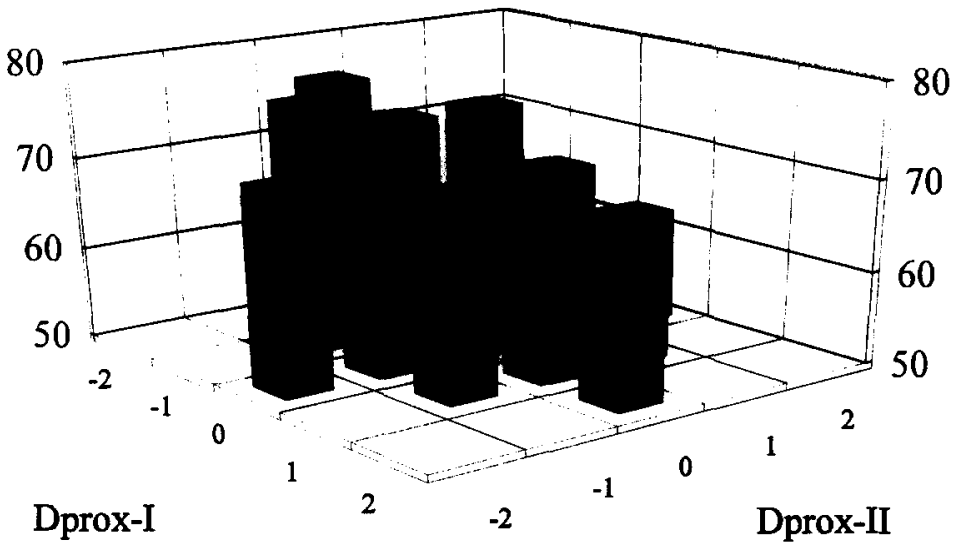

Fig. 13. Results from Experiment 3. The percentage of preference for the dominant colors (red and brown) are plotted as a function of the Dprox coordinates for all configurations. Dprox I refers to the relative position of Circles I, whereas Dprox II refers to the relative position of Circles II. It can be seen that, in general, the percentages are highest for negative Dprox coordinates.

of C10). In the same way, the height of the bar with Dprox coordinates $(1,1)$ reflects the judgements for the color of the corresponding segments of Configurations $\mathrm{C} 13$ and C14. Each of the bars of the graph of Fig. 13 belong to one of the three Stimulus Subsets of Fig. 12. The central bar for which the Dprox coordinates $(0,0)$ reflect the mean preferences for the dominant colors in Stimulus Subset 1, the bars with Dprox coordinates $(0,1),(0,-1),(1,0)$, and $(-1,0)$ reflect the mean preferences for the dominant colors in Stimulus Subset 2, and all other bars reflect the mean preferences for the dominant colors in Stimulus Subset 3.

Fig. 13 shows that negative values of Dprox I of a specific segment evoke the highest preferences for that segment, whereas positive values evoke the lowest preferences. An analysis of variance (Friedman ANOVA By Ranks) performed for all possible values of Dprox I (ranging from -2 to 2), calculated per subject for both colors, is highly significant: $X^{2}(4, N=16)=15.39, p<0.005$. The same analysis for all possible values of Dprox II (ranging from -2 to 2 ) is significant as well: $X^{2}(4, N=16)=9.53, p<0.05$. The latter result, however, is somewhat flattered by the percentages of preference for the extreme values of -2 and 2 for Dprox II. If the percentages of preference for the extreme values of -2 and 2, for both Dprox I and Dprox II are not included in the analysis, the results differ: The Friedman ANOVA By Ranks performed on Dprox I values ranging from -1 to 1 is still significant: $X^{2}(2, N=16)=6.00, p<0.05$, whereas the same analysis performed on Dprox II values ranging from -1 to 1 is no longer significant: $X^{2}(2, N=16)=1.56$ (n.s).

Indeed, inspecting the graph, a noticeable difference can be seen between the percentages for which Dprox I $=0$ and the percentages for which Dprox II $=0$. When Dprox $I=0$, the percentages of preference for Dprox $I I=-1$ and Dprox $I I=1$ are about equal, but differ greatly from the percentages for Dprox $I I=-2$ and Dprox 
$\mathrm{II}=2$, whereas when Dprox II $=0$, the percentages of preference for Dprox $\mathrm{I}=-1$ and Dprox $I=1$ differ greatly from each other, but are about equal to the percentages for Dprox II $=-2$ and Dprox II $=2$, respectively. As noted above, the bars for which the Dprox coordinates are $(1,0),(-1,0),(0,1)$ and $(0,-1)$, actually reflect the responses on the configurations of Stimulus Subset 2, in which one of two segments is regularly spaced. Because within this stimulus subset the color judgements might be influenced both by regularity and proximity, we take a closer look at these data.

\subsubsection{Regularity versus proximity}

In Fig. 14(a), the mean percentages of preference for Configurations C6 and C7 are plotted as a function of Regularity and Dprox I. In Fig. 14(b), the same has been done for Configurations C8 and C9, this time for Regularity and Dprox II. The regularity axis has two categories, Yes and No. Both the Dprox I axis and the Dprox II axes have two values, -1 and 1 . The height of the bars indicate the percentage of preference for the color of a specific segment within a configuration. For example, the height of the most left bar in Fig. 14(a) indicates the preference for the regular segment in Configuration C7. (Note that this is not necessarily the left segment as given in the drawing, because mirror versions were presented as well.)

We have separately analyzed the effects of Regularity versus Dprox I in Fig. 14(a) and Regularity versus Dprox II in Fig. 14(b). A Wilcoxon Matched Pairs Analyses By Ranks performed on the preferences between the two Regularity categories of Fig. 14(a) (pooling the preferences of the Dprox I values for each subject) yields: $z=1.98, p<0.05$. An analogous procedure with respect to the two Dprox I values yields: $z=1.96, p<0.05$. The preference between the two Regularity categories in Fig. 14(b) is significant as well $(z=2.67, p<0.01)$, whereas the difference between the two Dprox II categories is not significant $(z=1.31$, n.s.). So, whereas for the configurations in Fig. 14(a), both Regularity and Dprox I appear to play an essential role in the occurrence of color assimilation, for the configurations in Fig. 14(b), Regularity appears to be the only color inducing factor.

The competition between proximity and regularity can clearly be observed in the diagram of Fig. 14(a). Notice that whereas the position of Circle I seems to be of crucial influence on the preference for the color of the regular segment, the position of Circle II appears not to have such an influence (the latter replicating the result of Experiment 1). Further, for each of the configurations, the percentages of preference for both segments within a specific configuration have been compared with each other. The differences between these preferences again have been analyzed with a Wilcoxon Matched Pairs Analyses By Ranks on the percentages of preference for both colors per participant: Configuration C6: $z=0.52$ (n.s.); Configuration C7: $z=2.70$, $p<0.01$; Configuration C8: $z=2.47, p<0.05$; Configuration C9: $z=2.29, p<0.05$.

\section{General discussion}

The main findings of this study can be summarized as follows. First, the color of an element that is positioned such that it could belong to either of two neighboring 


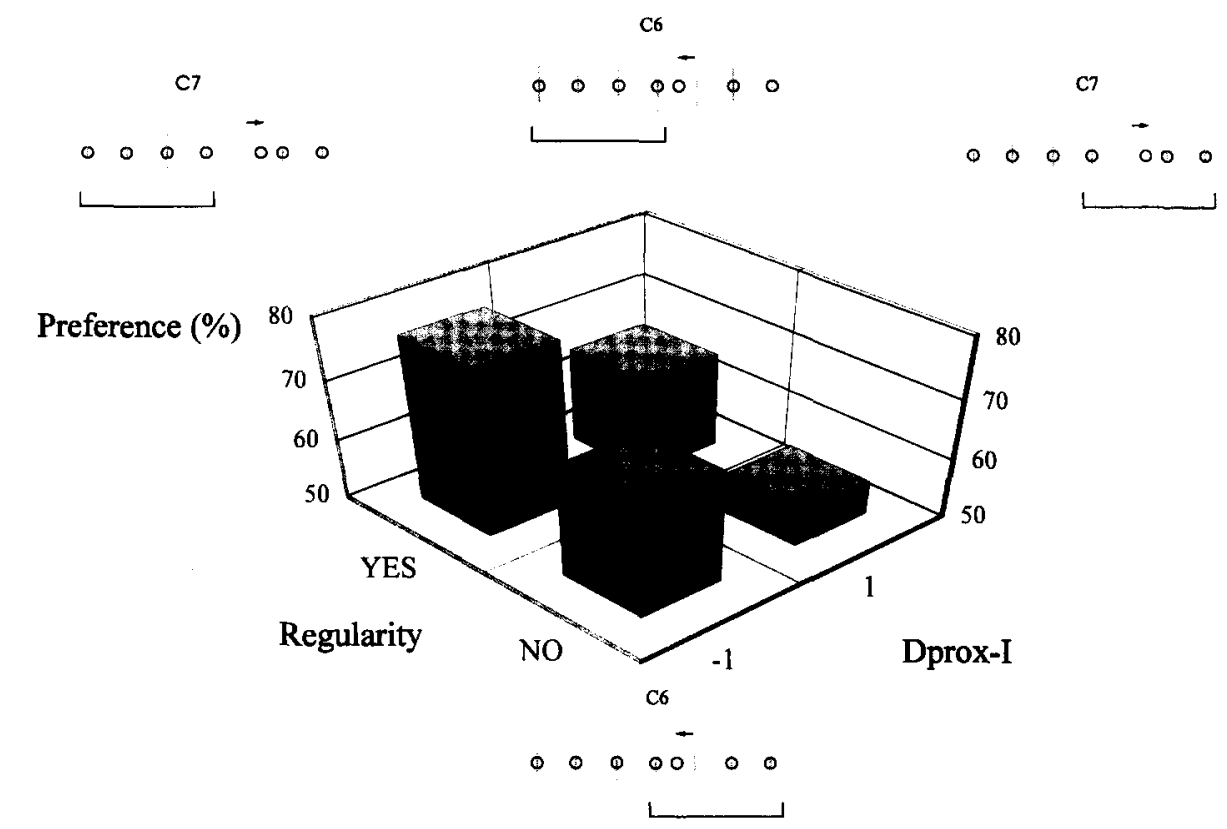

A

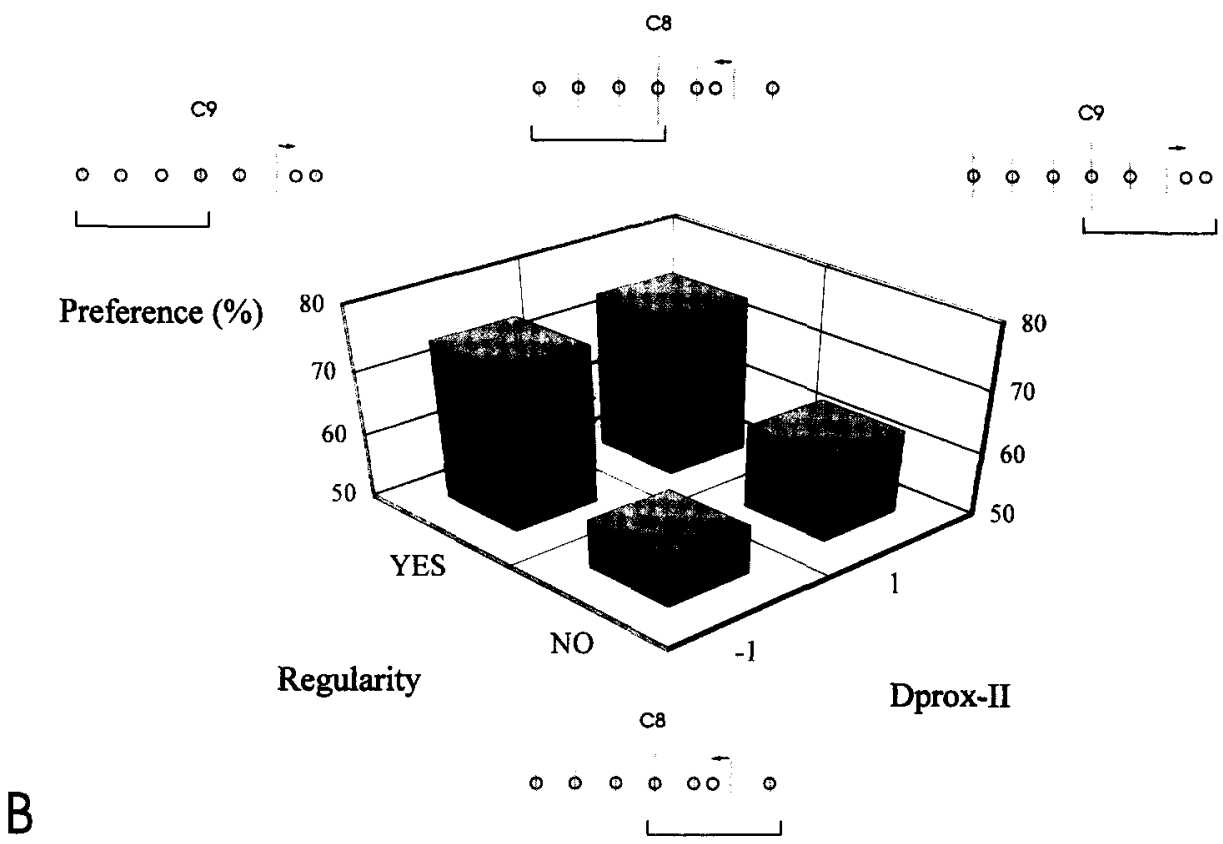

Fig. 14. Results from Experiment 3. In A, the percentages of preference for the dominant colors are plotted as a function of Regularity and Dprox I for each segment for Configurations C6 and C7 (for all segments with Dprox II =0). In $\mathrm{B}$, the percentages of preference for the dominant colors are plotted as a function of Regularity and Dprox II for each segment for Configurations C8 and C9 (for all segments with Dprox $I=0$ ). The height of the bars indicates the percentage of preference for the color of a specific segment within a configuration. 
collections of elements tends to look more like the color of the elements with which it can be grouped by means of regularity or proximity. Second, by measuring the strength of color assimilation, it is possible to compare the relative strengths of different groupings. In Experiment 1, color assimilation was induced by regularity for all five color pairs that were tested, although the effects were weaker for the color pairs with larger luminance differences. In Experiment 2, color assimilation was induced by proximity for only two of the five color pairs that were tested, whereas two other pairs showed trends towards contrast instead of assimilation. Again, this occurred for color pairs with larger luminance differences. To avoid a moderating effect of contrast and to better isolate assimilation effects, future research should preferably use isoluminant or near-isoluminant colors. In Experiment 3, where the stimulus configurations and color pairs allowed for both, grouping by regularity and grouping by proximity, we observed color assimilation effects of regularity and of proximity by the closest neighbor but a much weaker effect of proximity of the second nearest neighbor.

It is not easy to compare the relative strength of grouping by regularity and grouping by proximity. This can be illustrated most clearly by the results obtained with Configuration C6 in Experiment 3. Notice that although, on the whole, the preferences for either side of the configuration are about the same (i.e., not differing significantly), we cannot deduce from this result that for the given configuration, assimilation induced by proximity equals assimilation induced by regularity. With respect to such a comparison, we have to consider the possibility that, given present experimental conditions, the influences of proximity and regularity on color assimilation are unequal. If, for instance, for the given distances, proximity induces assimilation more strongly than regularity does, equal percentages for assimilation induction by proximity and regularity actually indicate a stronger relative influence of regularity.

In examining a turning point between the influences of regularity and proximity, the differences in induction strength must be taken into account. This can be done, to a certain extent, by comparing the preferences on both segments of Configuration C6 with the preferences on those configuration segments in which effects of either proximity or regularity can be regarded as baseline values. Given the present set of configurations, the assimilation induction by regularity in Configuration $\mathrm{C} 6$ can be estimated best by taking the mean preference for the regular segments in Configurations C8 and C9, having Dprox coordinates $(0,1)$ and $(0,-1)$, respectively (see Fig. 12). Note that these segments are exactly the same as the corresponding regular segment in Configuration C6. Analogously, the assimilation induction by proximity in Configuration $\mathrm{C} 6$ can be estimated best by the mean preferences on Configurations $\mathrm{C} 12$ and $\mathrm{C} 13$ for the segments with Dprox coordinates $(-1,+1)$ and $(-1,-1)$ (see Fig. 12). Note that these segments are exactly the same as the corresponding "proximity" segment in Configuration C6.

By doing so, the baseline percentage of preference on the assimilation by regularity is $(74.7 \%+75.1 \%) / 2=74.9 \%$, whereas the baseline percentage of preference on the assimilation by proximity is $(77.4 \%+78.6 \%) / 2=78 \%$. Regarding these baseline preferences, the net percentage of preference for the regular segment in Configura- 
tion $\mathrm{Cl}=65.9 \%-74.9 \%=-9 \%$, and the net percentage of preference for the proximity segment in Configuration $\mathrm{Cl}$ is $69 \%-78 \%=-9 \%$. So, accounting for the different induction strengths the small advantage for the proximate segment is nullified, somewhat coincidentally, gaining a perfect balance between the opposing assimilation inductions caused by regularity and proximity. Evidently, such turning points strongly depend on the relative distances between the local elements in the segments, but also on the characteristics of the regular segment. The determination of the turning points might enable us in future experiments to rank various regular configurations for their perceptual impact. In future research, the perceptual strength of various types of regularities would be considered to be tied to actual distances in the patterns. The results of the present experiments and the competition between proximity and regularity indicate that both metrical and structural aspects of patterns are crucial with respect to perceptual grouping, suggesting that any model on perceptual grouping sooner or later must incorporate both.

Of course, it is not a new statement to say that both metrical and structural aspects of patterns are important or that both proximity and regularity play a role in grouping. The importance of different grouping principles is also known from many other areas of perceptual organization such as, for example, visual occlusion and regularity detection, where similar issues occur under the more general heading of local and global factors. In fact, proximity is a typical local aspect of pattern organization, whereas regularity represents a more global aspect of pattern organization.

To illustrate the role of both local and global factors, let us first consider the case of visual occlusion. When the visual system is confronted with a configuration of two shapes that partially overlap, the partly occluded shape is almost always perceived to be complete behind the occluder and the form of the partly occluded shape is often well determined. How does the visual system determine one specific completion of the background shape amongst all different possibilities? According to the global minimum principle, the preferred solution is the one which is simplest for the pattern as a whole, or, in other words, the one with the maximal amount of regularity (e.g., Buffart et al., 1981). According to an alternative account, however, the completion of the background shape is mainly determined by the Gestalt law of good continuation (which is more local, in a sense): Patterns are completed in order to continue contour elements smoothly (e.g., Kellman and Shipley, 1991). Much recent research in the area of visual occlusion is directed at examining the role of these local and global factors (e.g., Boselie, 1988, 1994; Sekuler, 1994; Sekuler et al., 1994; Van Lier et al., 1994, 1995).

Another area of perceptual organization in which there is a long-standing issue of local versus global aspects is the above mentioned visual system's detection of regularities such as bilateral mirror symmetry (see Wagemans, 1995, for a review). For example, Julesz (1971) suggested that symmetry detection seems to operate at two different levels, depending on the type of pattern: For patterns with low spatial frequencies such as silhouette shapes, symmetry appears to be extracted globally, whereas a local point-by-point comparison seems to be required for patterns with high spatial frequencies such as random-dot patterns. Since then, many theories 
and models of symmetry detection have assumed that a fast, holistic process to select a potential symmetry axis is followed by a slow, point-by-point comparison process to evaluate it (e.g., Bruce and Morgan, 1975; Jenkins, 1983; Palmer and Hemenway, 1978). Much empirical work in the area of symmetry detection is directed at examining the relative contribution of local factors such as element positions and orientations and more global factors such as their spatial relations and the presence of dense clusters (e.g., Barlow and Reeves, 1979; Carmody et al., 1977; Locher and Wagemans, 1993; Wenderoth, 1996). More recently, models have also been developed to understand in more detail the emergence of a global symmetry percept from local element positions and orientations (e.g., Dakin and Watt, 1994; Labonté et al., 1995; Wagemans et al., 1993).

Although we do not claim that the present study has direct relevance to all of these important issues, we do believe that the development of a tool to assess the relative weight of local and global grouping principles or Gestalt laws such as proximity and regularity provides the necessary first step towards understanding their role in situations where they occur together. In our view, the present paradigm might very well be extended to other one-dimensional patterns. For example, it could be used to examine the relative weight of different groupings as predicted by different coding rules in SIT (being symmetry, repetition, and alternation; e.g., Van der Helm and Leeuwenberg, 1996) or perhaps even the effect of smooth continuation (e.g., Field et al., 1993; Kovács and Julesz, 1993). Furthermore, we envisage future research with an extension of the present paradigm to two-dimensional configurations where, for example, one can look for more subtle effects of the number of symmetry axes (e.g., by comparing triangular with rectangular or square configurations), collinearity and of closure (e.g., Elder and Zucker, 1993, 1994). For example, patterns as shown in Fig. 1 could be used as stimuli. If, in Fig. 1, D is considered as the Test Circle, the influence of different regular configurations of $\mathrm{A}, \mathrm{B}$ and $\mathrm{C}$, with varying axis of symmetry, on the perceptual grouping of D could be examined following the method outlined here. Obviously, a color setting has to be chosen for which assimilation occurs for both proximity and regularity.

In all kind of experimental designs, it will be interesting to look at both proximity and regularities in a systematic and controlled way. The present paper has laid the foundation for the further development of a tool that allows just that.

\section{Acknowledgements}

The authors thank Charles de Weert for his useful suggestions and for his assistance in the determination of the Chromaticity Coordinates and Luminances measured with equipment of the Nijmegen Institute of Cognition and Information (NICI). The authors also thank Michael Kubovy for his helpful remarks on a previous draft of this paper. This research was conducted at the Laboratory of Experimental Psychology at the University of Leuven, where the first author held a Visiting Postdoctoral Fellowship (NFWO). Financial support for this research from FWOVlaanderen to the second author $(\mathrm{G} .0210 .97 \mathrm{~N})$ is also gratefully acknowledged. 


\section{References}

Agostini, T., Proffitt, D., 1993. Perceptual organization evokes simultaneous lightness contrast. Perception $22,263-272$.

Barlow, H.B., Reeves, B.C., 1979. The versatility and absolute efficiency of detecting mirror symmetry in random dot displays. Vision Research 19, 783-793.

Benary, W., 1924. Beobachtungen zu einem Experiment über Helligkeitskontrast (Observations on an experiment about brightness contrast). Psychologische Forschung 5, 131-142.

Biederman, I., 1987. Recognition by components: A theory of human image understanding. Psychological Review 94, 115-147.

Boselie, F., 1988. Local versus global minima in visual pattern completion. Perception and Psychophysics $43,431-445$.

Boselie, F., 1994. Local and global factors in visual occlusion. Perception 23, 517-528.

Buffart, H., Leeuwenberg, E., Restle, F., 1981. Coding theory of visual pattern completion. Journal of Experimental Psychology: Human Perception and Performance 7, 241-274.

Carmody, D.P., Nodine, C.F., Locher, P.J., 1977. Global detection of symmetry. Perceptual and Motor Skills 45, 1267-1273.

Dakin, S.C., Watt, R.J., 1994. Detection of bilateral symmetry using spatial filters. Spatial Vision 8, 393 413.

DeValois, R., DeValois, K., 1988. Spatial Vision. Oxford Univ. Press, Oxford.

De Weert, Ch., 1991. Assimilation versus contrast. In: Valberg, A., Lee, B.B. (Eds.), From Pigments to Perception. Plenum, New York, pp. 305-311.

De Weert, Ch., Van Kruysbergen, N., 1987. Subjective contour strength and perceptual superimposition: Transparency as a special case. In: Petry, S., Meyer, G. (Eds.), The Perception of Illusory Contours. Springer, New York, pp. 165-170.

De Weert, Ch., Van Kruysbergen, N., 1997. Assimilation: Central and peripheral effects. Perception (in press).

Elder, J., Zucker, S., 1993. The effect of contour closure on the rapid discrimination of two-dimensional shapes. Vision Research 33, 981-991.

Elder, J., Zucker, S., 1994. A measure of closure. Vision Research 34, 3361-3369.

Festinger, L., Coren, S., Rivers, G., 1970. The effect of attention on brightness contrast and assimilation. American Journal of Psychology 83, 189-207.

Field, D.J., Hayes, A., Hess, R.F., 1993. Contour integration by the human visual system: Evidence for a local "association field". Vision Research 33, 173-193.

Fuchs, W., 1923. Experimentelle Untersuchungen über die Änderung von Farben unter dem Einfluss von Gestalten (Angleichungserscheinungen) (Experimental investigations on the alteration of color under the influence of Gestalten). Zeitschrift für Psychologie 92, 249-325.

Girgus, J., Rock, I., Egatz, R., 1977. The effect on the knowledge of reversibility on the reversibility of ambiguous figures. Perception and Psychophysics 22, 550-556.

Grossberg, S., Mingolla, E., 1985. Neural dynamics of form perception: Boundary completion, illusory figures, and neon color spreading. Psychological Review 92, 173-211.

Hochberg, J., McAlister, E., 1953. A quantitative approach to figural 'goodness'. Journal of Experimental Psychology 46, 361-364.

Jenkins, B., 1983. Component processes in the perception of bilaterally symmetric dot textures. Perception and Psychophysics 34, 433-440.

Julesz, B., 1971. Foundations of Cyclopean Perception. University of Chicago Press, Chicago.

Kanizsa, G., 1979. Organization in Vision: Essays on Gestalt Perception. Praeger, New York.

Kanizsa, G., 1988. Color and organization: A response to King. New Ideas in Psychology 6, 289-291.

Kellman, P.J., Shipley, T.F., 1991. A theory of visual interpolation in object perception. Cognitive Psychology 23, 141-221.

King, D., 1988. Assimilation is due to one perceived whole and contrast is due to two perceived wholes. New Ideas in Psychology 6, 277-288.

Koffka, K., 1935. Principles of Gestalt Psychology. Harcourt, Brace and World, New York. 
Kovács, I., Julesz, B., 1993. A closed curve is much more than an incomplete one: Effect of closure in figure-ground segmentation. Proceedings of the National Academy of Sciences of the USA, vol. 90, pp. $7495-7497$.

Köhler, W., 1920. Die Physischen Gestalten in Ruhe und im stationären Zustand (Physical Gestalten in Rest and in Stationary State). Vieweg, Braunschweig.

Kubovy, M., 1994. The perceptual organization of dot lattices. Psychonomic Bulletin and Review 1, 182 190.

Kubovy, M., Holcombe, A., Wagemans, J., 1997. Grouping by proximity in periodic dot patterns shows no effect of configuration. (Previous version available as Intern. Rep. No. 196). University of Leuven, Laboratory of Experimental Psychology (submitted for publication).

Kubovy, M., Wagemans, J., 1995. Grouping by proximity and multistability in dot lattices: A quantitative Gestalt Theory. Psychological Science 6, 225-234.

Labonté, F., Shapira, Y., Cohen, P., Faubert, J., 1995. A model for global symmetry detection in dense images. Spatial Vision 9, 33-55.

Leeuwenberg, E., 1969. Quantitative specification of information in sequential patterns. Psychological Review 76, 216-220.

Locher, P., Wagemans, J., 1993. Effects of element type and spatial grouping on symmetry detection. Perception 22, 565-587.

Lowe, D.G., 1985. Perceptual Organization and Visual Recognition (Ph.D. Thesis, Department of Computer Science, Stanford University). Kluwer Academic Publishers, Boston, MA.

Metelli, F., 1967. Zur Analyse der phänomenalen Durchsichtigkeitserscheinungen (Analysis of phenomenal transparency). In: Mühlher, R., Fischl, J. (Eds.), Gestalt und Wirklichkeit. Dunker und Humboldt, Berlin.

Musatti, C., 1931. Forma e assimiliazone [Form and assimilation]. Archivo Italiano di Psychologica 9 , 213-269.

Oyama, T., 1961. Perceptual grouping as a function of proximity. Perceptual and Motor Skills 13, 305306.

Palmer, S.E., Hemenway, K., 1978. Orientation and symmetry: Effects of multiple, rotational, and near symmetries. Journal of Experimental Psychology: Human Perception and Performance 4, 691-702.

Palmer, S., Rock, I., 1994. Rethinking perceptual organization: The role of uniform connectedness. Psychonomic Bulletin and Review 1, 29-55.

Rock, I., Mitchener, K., 1992. Further evidence of failure of reversal of ambiguous figures by uninformed subjects. Perception 21, 39-45.

Sekuler, A.B., 1994. Local and global minima in visual completion: Effects of symmetry and orientation. Perception 23, 529-545.

Sekuler, A.B., Palmer, S.E., Flynn, C., 1994. Local and global processes in visual completion. Psychological Science 5, 260-267.

Stalmeier, P., De Weert, , Ch.1988. Large colour differences measured by spontaneous gestalt formation. Color Research and Application 13, 209-217.

Van der Helm, P., Leeuwenberg, E., 1991. Accessibility, a criterion for regularity and hierarchy in visual pattern codes. Journal of Mathematical Psychology 35, 151-213.

Van der Helm, P., Leeuwenberg, E., 1996. Goodness of visual regularities: A non-transformational approach. Psychological Review 103, 429-456.

Van der Helm, P., Van Lier, R., Leeuwenberg, E., 1992. Serial pattern complexity: Irregularity and hierarchy. Perception 21, 517-544.

Van Lier, R., 1996. Simplicity of visual shape. Ph.D. Thesis, University of Nijmegen.

Van Lier, R., Van der Helm, P., Leeuwenberg, E., 1994. Integrating global and local aspects of visual occlusion. Perception 23, 883-903.

Van Lier, R., Van der Helm, P., Leeuwenberg, E., 1995. Competing global and local completions in visual occlusion. Journal of Experimental Psychology: Human Perception and Performance 21, 571-583.

Van Oeffelen, M., Vos, P., 1983. An algorithm for pattern description on the level of relative proximity. Pattern Recognition 26, 341-348.

Von Bezold, W., 1876. The Theory of Color. Prang, Boston. 
Wagemans, J., 1995. Detection of visual symmetries. Spatial Vision 9, 9-32.

Wagemans, J., Van Gool, L., Swinnen, V., Van Horebeek, J., 1993. Higher-order structure in regularity detection. Vision Research 33, 1067-1088.

Wenderoth, P., 1996. The effect of dot pattern parameters and constraints on the relative salience of vertical bilateral symmetry. Vision Research 36, 2311-2320.

Wertheimer, M., 1923. Untersuchungen zur Lehre von der Gestalt (Investigations for the use of the study of Gestalt). Psychologische Forschung 4, 301-350.

Wittebrood, J., Wansink, M., De Weert, , Ch.1981. A versatile Color Stimulus Generator. Perception 10, 63-69. 